\section{P40 A META-FRAMEWORK FOR INCORPORATING SOCIO- ECONOMIC HEALTH INEQUALITIES IN SYSTEMATIC REVIEWS}

M Maden* . Liverpool Reviews and Implementation Group, University of Liverpool, Liverpool, UK

\subsection{6/jech-2018-SSMabstracts. 166}

Background Recent equity systematic review guidance encourages reviewers to consider whether it is likely that their findings may impact on health inequalities. Much of the guidance assumes that health inequalities have either already been identified as the focus of the review, or that reviewers are able to recognise if and how health inequalities matter. However, our experience is that this is not necessarily true. Furthermore, theorising if and how health inequalities matter is not normally integrated into the systematic review process. This presentation describes a novel approach to the development of a theory-led meta-framework to inform socio-economic health inequality considerations in systematic reviews.

Methods Following the best-fit framework synthesis approach, a meta-framework was generated by 'deconstituting' concepts from theories relating to complex interventions and socio-economic health inequalities into a single framework. Theories were identified via; i) searches in MEDLINE, CINAHL, The Cochrane Library (CDSR, Other reviews, HTA), the Database of Promoting Health Effectiveness Reviews (DoPHER), the Campbell Collaboration Library of Systematic Reviews, 3ie (International Initiative for Impact Evaluation) database of systematic reviews, Google Scholar, Campbell and Cochrane Equity Methods Group website), ii) scanning of known relevant theoretical papers, iii) theories identified in a published work on the use of programme theory in socio-economic focused systematic reviews and iv) informal discussions with health inequality experts. Feedback was sought from health inequality experts and reviewers.

Results Four complex intervention theories identify four domains and key factors that may influence effectiveness; intervention design, implementation, context and participant response. Applying an equity lens, 16 socio-economic health inequality theories identify key factors and mechanisms associated with these domains that may lead to differential effects across disadvantaged populations.

Conclusion The meta-framework has the potential to i) facilitate the identification and understanding of when, why and how intervention effectiveness may be moderated by socio-economic status, ii) promote a theory-led approach to incorporating socio-economic health inequality considerations in systematic reviews iii) help reviewers identify the type of data to extract and inform a priori analysis on what factors are associated with differential effects across socio-economic groups, iv) help reviewers to decide whether it is likely that their review findings may have the potential for an intervention to indirectly widen or narrow socio-economic health inequalities, even when evidence of an impact in the primary research is lacking. The meta-framework aims to increase the usefulness of systematic reviews in informing and implementing changes to practice.

\section{\begin{tabular}{|l|l}
\hline P41 ASSESSMENT OF PUBLICATION BIAS IN SYSTEMATIC \\
\hline
\end{tabular} REVIEWS OF HEALTH SERVICES AND DELIVERY RESEARCH}

${ }^{1}$ AA Ayorinde*, ${ }^{2}$ I Williams, ${ }^{2} \mathrm{R}$ Mannion, ${ }^{3} \mathrm{~F}$ Song, ${ }^{4} \mathrm{M}$ Skrybant, ${ }^{1} \mathrm{RJ}$ Lilford, ${ }^{1} \mathrm{Y}$ - $\mathrm{F}$ Chen. 'Warwick Centre for Applied Health Research and Delivery, University of Warwick, Coventry, UK; ${ }^{2}$ Health Services Management Centre, University of Birmingham, Birmingham, UK; ${ }^{3}$ Department of Population Health and Primary Care, University of East Anglia, Norwich, UK; ${ }^{4}$ Institute of Applied Health Research, University of Birmingham, Birmingham, UK

\subsection{6/jech-2018-SSMabstracts. 167}

Background Publication bias is a major threat to the validity of systematic reviews (SRs). Strategies to identify and reduce publication bias are routinely incorporated into SRs of clinical interventions, but the level of adoption of these strategies in SRs relating to health services and delivery research (HSDR) is unclear. The objectives of this study were to describe the characteristics of SRs of HSDR with regards to assessment of publication bias, and to evaluate factors associated with this.

Methods A stratified random sample of 200 SRs of quantitative HSDR published in English from 2007-2017 was selected from the Health Systems Evidence database. Half (100) of the selected reviews (intervention reviews) concern interventions to improve the effectiveness/efficiency of service delivery, which would mostly involve comparative studies. The other half (association reviews) evaluates associations between different variables along the service delivery causal chain, which often include observational studies. Data extracted included: any reference to publication bias, methods for detecting/mitigating publication bias or reasons for no assessment, number of included studies, inclusion of meta-analyses and whether the use of SR guideline was reported. Journals were divided into those that did or did not formally endorse specific SR guidelines such as PRISMA, and journal impact factors were obtained. Factors associated with assessment of publication bias were explored using multivariable logistic regression.

Results Of all 200 SRs, 48\% commented on publication bias. However, only $25 \%$ formally assessed publication bias either through statistical analysis (mostly funnel plots) or as part of the quality assessment of included studies (e.g. the Cochrane risk of bias tools). Insufficient number of studies, heterogeneity and lack of pre-registered protocols were common impediments in assessing publication bias. In the multivariable analysis, assessment of publication bias was associated with SRs' inclusion of a meta-analysis [odds ratio (OR) 9.08 (95\% CI 3.67, 22.43)], reviewers reporting the use of SR guidelines [OR 4.09 (1.71, 9.78)], being an intervention review [OR $3.75(1.42,9.91)]$ and higher journal impact factor [OR 1.16 $(1.02,1.30)]$ but not significantly associated with number of studies included in SRs or journal endorsement of SR guidelines.

Conclusion Overall, the awareness of publication bias in HSDR reviews is comparable to that of reviews of clinical interventions, however, formal assessment of publication bias is less common especially in association reviews. This reflects the heterogeneity of HSDR evidence and the limits of current tools for assessing publication bias. Adherence to existing SR guidelines should be promoted. 